\title{
The Relationship between Blood Viscosity and Acute Arterial Occlusion
}

\author{
Güney Erdoğan, Mustafa Yenerçağ, Uğur Arslan \\ Department of Cardiology, Training and Research Hospital, University of Health Sciences, Samsun, Turkey
}

\section{ABSTRACT}

Background: Blood viscosity is a strong predictor of cardiovascular events. However, the relationship between blood viscosity and acute arterial occlusion (AAO) has not been studied adequately so far. Objectives: The aim of the present study was to assess the relationship between whole blood viscosity (WBV) and AAO. Material and methods: The study included 93 patients who were diagnosed with AAO between January 2017 and September 2019, and 90 age- and sex-matched healthy controls. WBV was assessed using a validated calculation formula derived from hematocrit and total plasma protein levels, both as a low (LSR) and a high (HSR) shear rate. Results: There were no significant differences between the two groups in regards to the baseline characteristics, with the exception of smoking and LDL cholesterol levels. Subjects with AAO presented significantly higher WBV values both at LSR $(32.2 \pm 5.0$ vs. $26.6 \pm 5.0, \mathrm{p}<0.001)$ and HSR $(6.2 \pm 0.3$ vs. $5.7 \pm 0.3, \mathrm{p}<0.001)$. The ROC analysis revealed a cut-off value of 27.4 for WBV at LSR (sensitivity $66 \%$, specificity $64 \%$, AUC $=0.770, p<0.001$ ) and a cut-off value of 5.29 in case of HSR (sensitivity $69 \%$, specificity 74\%, AUC = 0.801, p $<0.001$ ) for predicting AAO. Multivariate analysis, both LSR (OR 3.33, 95\% CI: 1.20-9.43, p = 0.006 ) and HSR ( $\mathrm{p}=0.020$, OR: 1.70, 95\% CI: 1.020-1.123) were independent predictors of AAO. Conclusions: This study demonstrated that WBV levels at both HSR and LSR are significantly higher in the AAO group than in the control subjects, indicating that an increased WBV may be associated with the development of AAO.

Keywords: acute arterial occlusion, whole blood viscosity, peripheral artery disease

\section{ARTICLE HISTORY}

Received: February 1, 2020

Accepted: March 5, 2020

\section{CORRESPONDENCE}

Uğur Arslan

Baris Bulvarı No: 199

Ilkadim/Samsun, Turkey

Tel: +90 5326039983

E-mail: ugurarslan5@yahoo.com

\section{INTRODUCTION}

Acute arterial occlusion (AAO), also known as acute limb ischemia, is a vascular emergency requiring urgent diagnosis and treatment. This condition is characterized by a loss of perfusion in a peripheral artery, either in the upper or lower extremities, within 2 weeks after the initiating event. ${ }^{1}$ The most common cause of this condition is represented by in situ thrombotic obstructions and thromboembolism. While in situ thrombotic obstructions occur as a result of plaque rupture due to pre-existing peripheral arterial disease, embolic obstructions consist of thromboembolic processes originating from the heart or from the aortoiliac vessels. ${ }^{2}$ In situ thrombosis most commonly affects the femoral or popliteal arteries, while emboli most commonly lodge in the aortic, iliac, femoral, and popliteal bifurcations. ${ }^{3}$ Despite medical and surgical treatments, AAO is still linked to an increased morbidity and mortality.4,5

Hemorheological factors have a significant contribution in the atherosclerotic process. ${ }^{6}$ A relationship between 
conventional atherosclerosis risk factors and blood viscosity has been shown in many studies. Increased blood viscosity results in intravenous stasis and endothelial dysfunction, contributing to thrombus formation. Eventually, blood viscosity plays a key role in the formation of an atherothrombotic environment. ${ }^{7}$ Whole blood viscosity (WBV) can be measured using a validated equation from hematocrit and total plasma protein levels according to low and high shear rate by routine laboratory measurements. ${ }^{8}$

In this context, we aimed to investigate the possible relationship between WBV and AAO.

\section{METHODS}

This cross-sectional, single-center, retrospective study included AAO patients who were treated either medically or percutaneously with catheter-based treatments or surgically between January 2017 and September 2019 in the Samsun Training and Research Hospital. For the diagnosis of AAO, a detailed physical examination was done for changes along the extremity affected with a bilateral comparison. Then, patients with deficient pulses underwent bilateral lower limb arterial color Doppler ultrasonography with a handheld device in the emergency unit. When occlusion was detected, confirmatory testing with computed tomography angiography (CTA) was performed as quickly as possible. Patients with anticoagulant drug use for any reason, acute coronary syndromes, important hypertrophy of the left ventricle (septal thickness $>13 \mathrm{~mm}$ ), severe valvular disorders (including patients treated surgically or percutaneously), chronic infectious diseases, chronic heart failure, chronic inflammatory or autoimmune diseases, and oncologic pathologies were excluded. The reason for excluding these patients was the relationship between these conditions and an increased inflammation, which may affect WBV and therefore our results. Ninety-three subjects with AAO, over 18 years old were included in the study, and 90 healthy age- and gendermatched subjects were selected as controls. Demographical data, baseline cardiovascular risk factors, medical history, drugs used, and laboratory values were obtained using the hospital's medical database.

The ejection fraction of the left ventricle was assessed with the modified Simpson method, using 2D transthoracic echocardiography (Vivid 9, GE Ving Med Ultrasound, Horten, Norway). Peripheral blood samples were obtained after a 12-hour overnight fasting. Dry tubes for biochemical tests and tubes with ethylenediaminetetraacetic acid (EDTA) for hematological tests were used. The biochemical measurements were performed using a molecular analyzer
(Roche Diagnostics, Manheim, Germany). WBV was calculated consistent with de Simone's formula, ${ }^{8}$ at both high shear rate $\left(\mathrm{HSR}=208 / \mathrm{sec}^{-1}\right)$ and low shear rate $(\mathrm{LSR}=0.5 /$ $\mathrm{sec}^{-1}$ ). All data were compared between the two groups. The research procedures were revised and approved by the local hospital's ethics committee, according to the ethical considerations stipulated in the Helsinki Declaration.

\section{WHOLE BLOOD VISCOSITY MEASUREMENT}

WBV was calculated according to de Simone's formula, using blood tests conducted during hospital admission (whole blood count and biochemical values). WBV was calculated using hematocrit (Hct), and total protein (TP) values. ${ }^{8}$

$$
\begin{aligned}
& \text { WBV at HSR }=(0.12 \times \mathrm{HCT})+0.17(\mathrm{TP}-2.07) \\
& \text { WBV at } \mathrm{LSR}=(1.89 \times \mathrm{HCT})+3.76(\mathrm{TP}-78.42)
\end{aligned}
$$

\section{STATISTICAL ANALYSIS}

Statistical analysis was performed using Statistical Package for Social Sciences (SPSS) version 23 (IBM Corp., Armonk, NY, USA). The analysis of independent samples was performed using Student's t test for comparison of quantitative data with normal distribution and the MannWhitney U test for data with non-parametric distribution. Categorical data were analyzed by the chi-square test. We performed a univariate analysis on different factors associated with AAO. Variables in which the unadjusted pvalue had reached statistical significance $(p<0.05)$ were included in the full multivariate models. For WBV at HSR and LSR, two different models were designed: WBV + HSR was incorporated in Model 1 and WBV + LSR in Model 2. Independent risk factors affecting outcome were calculated by multivariate logistic regression analysis and comparative results were presented. The best cut-off value for both LSR and HSR regarding clinical outcome was examined by receiver operating characteristic (ROC) analysis, and area under the curve (AUC), sensitivity, specificity, positive predictive value, negative predictive value, and accuracy values corresponding to each cut-off value were presented. Odds ratios (ORs) and 95\% confidence intervals were calculated; sensitivity and specificity values for outcome classification were generated. The statistical significance level was accepted as $\mathrm{p}<0.05$.

\section{RESULTS}

The comparisons of baseline characteristics and laboratory findings between groups are presented in Table 1 . There 
TABLE 1. Baseline characteristics and laboratory findings of the study population

\begin{tabular}{lccc}
\hline Parameters & $\begin{array}{c}\text { AAO patients } \\
(\mathbf{n}=\mathbf{9 3})\end{array}$ & $\begin{array}{c}\text { Control patients } \\
(\mathbf{n}=\mathbf{9 0})\end{array}$ & p value \\
\hline Age, years (mean $\pm \mathrm{SD})$ & $72.0 \pm 9.8$ & $70.7 \pm 2.7$ & 0.320 \\
Men, n (\%) & $50(53.8)$ & $44(48.9)$ & 0.290 \\
HTN, n (\%) & $42(45.2)$ & $44(48.9)$ & 0.710 \\
DM, n (\%) & $31(33.3)$ & $28(31.1)$ & 0.030 \\
Atrial fibrillation, n (\%) & $24(25.8)$ & $19(21.1)$ & 0.060 \\
Smoking, n (\%) & $46(49.4)$ & $26(28.9)$ & 0.015 \\
BMI (kg/m $\left.{ }^{2}\right)$ & $27.4 \pm 5.0$ & $26.5 \pm 3.7$ & 0.250 \\
LDL-C (mg/dL) & $118.1 \pm 30.1$ & $106.0 \pm 27.7$ & 0.020 \\
Triglyceride (mg/dL) & $148.7 \pm 68.5$ & $141.3 \pm 84.2$ & 0.560 \\
Hemoglobin (g/dL) & $14.2 \pm 0.9$ & $13.8 \pm 0.7$ & 0.091 \\
Hematocrit (\%) & $42.7 \pm 2.7$ & $41.5 \pm 2.3$ & 0.102 \\
Total protein (g/L) & $7.3 \pm 0.6$ & $7.0 \pm 0.8$ & 0.088 \\
Platelets (103) & $259.8 \pm 90.0$ & $249.5 \pm 64.0$ & 0.470 \\
Creatinine (mg/dL) & $1.2 \pm 0.9$ & $1.1 \pm 0.8$ & 0.410 \\
LVEF (\%) & $56.7 \pm 4.4$ & $56.4 \pm 5.1$ & 0.702 \\
\hline
\end{tabular}

Data are given as mean $\pm \mathrm{SD}$, median (interquartile range) or $\mathrm{n}(\%)$.

AAO - acute arterial occlusion; DM - diabetes mellitus; HTN - hypertension; HPL - hyperlipidemia; BMI - body mass index; LDL-C - low-density lipoprotein cholesterol; HDL-C - high-density lipoprotein cholesterol; LVEF left ventricular ejection fraction

were no significant differences between the two groups in terms of baseline demographic characteristics except the prevalence of smoking ( $46 \%$ vs. $26 \%$; $=0.015$ ) and low-density lipoprotein cholesterol (LDL-C) (118.1 \pm 30.1 $\mathrm{mg} / \mathrm{dL}$ vs. $106.0 \pm 27.7 \mathrm{mg} / \mathrm{dL}, \mathrm{p}=0.020$ ) (Table 1). AAO patients had significantly higher WBV values both at LSR (32.2 \pm 5.0 vs. $26.6 \pm 5.0, \mathrm{p}<0.001)$ and HSR $(6.2 \pm 0.3$ vs. $5.7 \pm 0.3, \mathrm{p}<0.001$ ) (Figure 1 ).

The univariate analysis identified several variables for predicting AAO including: active smoking, diabetes, atrial fibrillation, LDL-C, WBV at HSR, and WBV at LSR ( $\mathrm{p}<0.05)$ (Table 2). We designed two models derived from a multi- variate analysis on the predictors of WBV parameters for each shear rate, which are illustrated in Table 3. In Model 1, which described WBV at HSR, the results showed that smoking, diabetes, high LDL-C levels, and WBV at HSR were independent predictors of AAO. In the second model (WBV at LSR), smoking, diabetes mellitus, LDL-C levels, and WBV at LSR were also found to independently predict AAO. In both models, WBV was an independent predictor of AAO ( $\mathrm{p}<0.05)$ (Table 3).

The ROC analysis revealed that a cut-off value of 27.4 for WBV at LSR had a $66 \%$ sensitivity and $64 \%$ specificity (AUC: $0.770, p<0.001$ ), while a cut-off value of 5.92 for
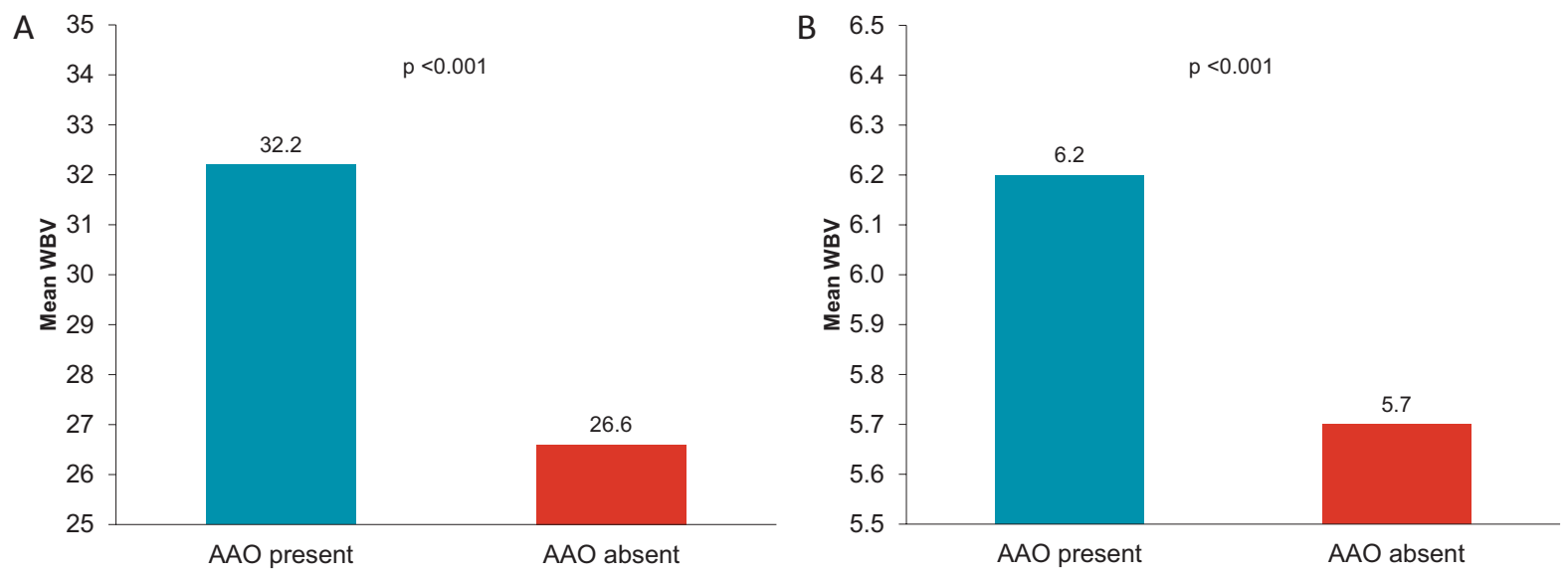

FIGURE 1. A - WBV levels at LSR in both groups; $\mathbf{B}-$ WBV levels at HSR in both groups 
TABLE 2. Univariate logistic regression analysis

\begin{tabular}{lccc}
\hline Variables & OR & $\mathbf{9 5 \%}$ CI & p value \\
\hline LDL-C (mg/dL) & 1.014 & $1.002-1.026$ & 0.020 \\
Smoking & 2.965 & $1.406-6250$ & 0.004 \\
DM & 2.150 & $1.043-4.432$ & 0.038 \\
Atrial fibrillation & 2.502 & $1.235-5.064$ & 0.011 \\
WBV at LSR, 208 s & 1.082 & $1.032-1.134$ & 0.001 \\
WBV at HSR, 0.5 s & 4.552 & $1.591-13.030$ & 0.005 \\
\hline
\end{tabular}

LDL-C - low-density lipoprotein cholesterol; DM - diabetes mellitus; WBV - whole blood viscosity; LSR - low shear rate; HSR - high shear rate; OR - odds ratio; CI - confidence interval

WBV at HSR had a $69 \%$ sensitivity and $74 \%$ specificity for predicting of AAO in the study population (AUC: $0.801, p$ $<0.001$ ) (Figure 2).

\section{DISCUSSIONS}

To our knowledge, this is the first study showing that WBV values at both low and high shear rates are more increased in patients with AAO compared to healthy controls. Furthermore, WBV at both at LSR and HSR was found to be an independent predictor of AAO.

AAO results from the acute occlusion of a peripheral artery or a bypass graft, causing a sudden decrease in blood flow in the region irrigated by the occluded artery or graft. Risk factors for AAO include age, smoking, diabetes mellitus, obesity, sedentary lifestyle, familial vascular disease history, high cholesterol and blood pressure. ${ }^{9}$ Arterial thrombosis causes $85 \%$ of AAOs, while embolic events are the cause of AAO in $15 \%$ of cases. Thrombosis can occur when chronic stenotic lesions in occlusive atherosclerosis lead to acute obstruction, usually associated with a plaque breakdown, circulatory failure, stasis, or a hypercoagulable state. Cardiogenic emboli trigger around $90 \%$ of peripheral arterial embolism and can occur secondary to atrial fibrillation, valvular heart disease, or myocardial infarction. In rare cases, cardiogenic em- boli are associated with paradoxical embolism through a patent foramen ovale. ${ }^{10}$ Consequently, atherosclerosis and thrombosis play a major role in the pathophysiology of AAO.

The main determining factors of blood viscosity are represented by plasma viscosity, hematocrit, red cell deformation, and aggregation. ${ }^{11}$ Measurement and evaluation of blood viscosity can be difficult and complex in daily practice. De Simone et al. calculated WBV by simple equations using hematocrit and total protein levels at different shear rates, indicative of different hemodynamic environments. ${ }^{8}$ They supported the accuracy of this formula by conducting validation analyses with the Viscometer device, an instrument used to measure blood viscosity. WBV calculated with this formula was evaluated in several cardiovascular disorders including myocardial infarction, heart failure, and coronary slow flow phenomenon. ${ }^{12-14}$ Consequently, we decided to determine WBV using the formula of de Simone at both shear rates, being less expensive and more simple in our study.

Blood viscosity is the inherent resistance of the blood stream in the vasculature and is closely related to blood flow rate. An abnormal blood viscosity is associated with decreased tissue perfusion and with the development of atherosclerosis. As a result of increased blood viscosity, stasis occurs secondary to the deterioration of blood flow

TABLE 3. Predictors of $A A O$ in multivariate logistic regression analyses

\begin{tabular}{lccccccc}
\hline MODEL 1 & \multicolumn{7}{c}{ MODEL 2 } \\
\hline Variables & OR & $\mathbf{9 5 \%} \mathbf{~ C I}$ & p value & Variables & OR & $\mathbf{9 5 \% ~ C I ~}$ & p value \\
\hline LDL-C (mg/dL) & 1.015 & $1.001-1.029$ & 0.030 & LDL-C (mg/dL) & 1.015 & $1.001-1.029$ & 0.026 \\
Smoking & 2.66 & $1.181-6.000$ & 0.018 & Smoking & 2.46 & $1.089-5.555$ & 0.030 \\
DM & 1.76 & $0.805-3.806$ & 0.150 & DM & 1.94 & $0.885-4.269$ & 0.098 \\
WBV at HSR & 1.070 & $1.020-1.123$ & 0.020 & WBV at HSR & 3.377 & $1.209-9.431$ & 0.006 \\
\hline
\end{tabular}

AAO - acute arterial occlusion; DM - diabetes mellitus; LDL-C - low-density lipoprotein cholesterol; WBV - whole blood viscosity; LSR - low shear rate; HSR - high shear rate; OR - odds ratio; CI - confidence interval 


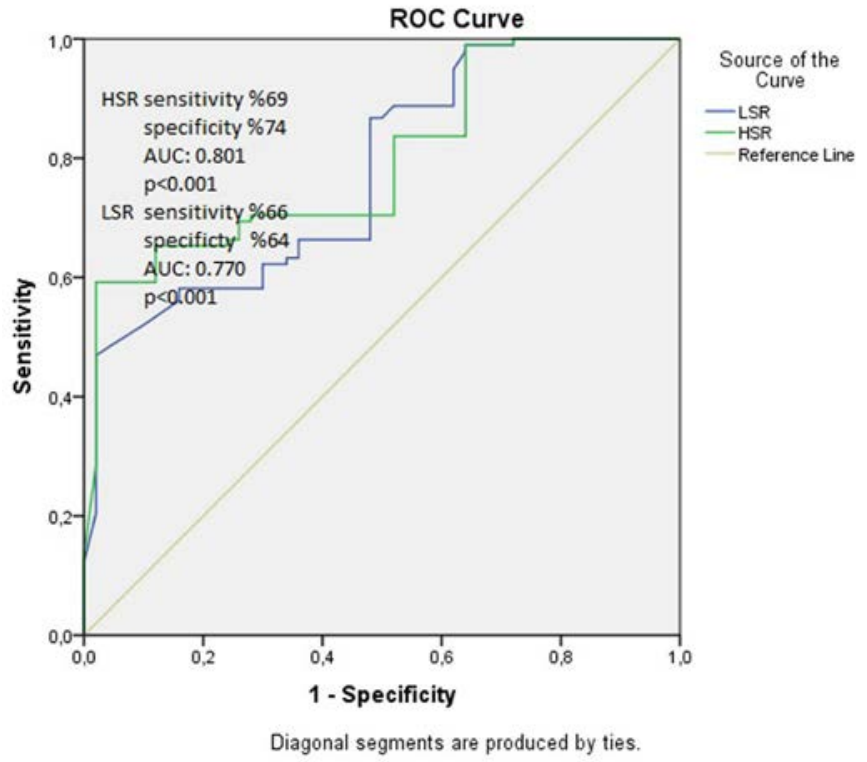

FIGURE 2. ROC curve analysis showing the predictive cut-off value of WBV at a high shear rate (blue line) and low shear rate (green line) for AAO patients. AUC - area under the curve; HSR high shear rate; LSR - low shear rate; ROC - receiver operating characteristic; WBV - whole blood viscosity.

rate. The disruption of laminar flow state creates turbulent blood flow. Turbulent flow triggers the disruption of the surrounding endothelial integrity, ${ }^{15}$ and also contributes to the blocking of platelet aggregates in small vessels. ${ }^{16}$ All these pathological steps increase the tendency of both arterial and venous thrombosis. Some studies have demonstrated that WBV correlates with retinal vessel thrombosis. ${ }^{17}$ Gunes et al. found that elevated WBV was an important and independent risk factor in patients with deep vein thrombosis. ${ }^{18}$ In our study, we demonstrated that increased WBV values are independent risk factors for AAO formation. These results support the view that elevated WBV may be an important risk factor for thrombus formation.

An increased blood viscosity may lead to an impaired tissue level perfusion and is frequently involved in the development and progression of atherosclerosis. WBV is one of the most important determinants of endothelial shear stress (ESS). A change in blood viscosity may cause low ESS, thus contributing to the initiation of the atherosclerotic process at the endothelial level. This process leads to an increased tendency for thrombosis with low-density lipoprotein deposition due to high blood viscosity in low flow regions caused by atheroma formation. ${ }^{7,19}$ In summary, the etiology of most AAOs is thrombosis and atherosclerosis, which are associated with increased blood viscosity. Studies supporting this hypothesis documented the relationship between hemorheological parameters and carotid or coronary artery atherosclerosis. ${ }^{20-22}$ In addition, some studies have shown that increased WBV is associated with high morbidity and mortality in some settings, including vascular diseases. ${ }^{23}$

Inflammation is one of the key drivers of atherosclerotic plaque development. At the same time, one of the hallmarks of atherosclerotic disease is represented by the interplay between inflammation and lipids. Risk factors for atherosclerosis also contribute to the development of this inflammatory process. In a study by Saskın et al., inflammatory markers were found to be increased in patients with $\mathrm{AAO}^{24}$ In addition, after the development of $\mathrm{AAO}$, inflammatory mediators may also increase due to tissue necrosis, similarly to acute myocardial infarction. Increased inflammation in AAO patients may also contribute to the elevation of blood viscosity, which should be investigated in further studies.

Several earlier studies have demonstrated that a higher blood viscosity is linked to an increase in the incidence of various cardiovascular disorders. In their study, Lowe et al. have shown that plasma WBV was correlated with a higher prevalence of cardiovascular risk factors, as well as with significant coronary and peripheral atherosclerosis. ${ }^{25}$ Furthermore, in the PIVUS (Prospective Investigation of the Vasculature in Uppsala Seniors) study, WBV was positively related to the Framingham Risk Score. ${ }^{26}$ These studies show that blood viscosity may be counted as a cardiovascular risk factor. In our study, we found increased WBV values in AAO patients, which also supports these results. Consequently, our study has shown that increased WBV was associated with the development of AAO.

\section{LIMITATIONS}

Our study has some limitations. Intrinsic limitations due to the retrospective, single-center design of the study should not be ignored. Furthermore, we did not measure WBV directly, which is the current gold standard technique for the assessment of blood viscosity; instead, we used a formula, which was clinically approved in many studies, for the calculation of WBV.

\section{CONCLUSIONS}

To the best of our knowledge, this is the first study to show that WBV levels at both HSR and LSR were significantly higher in AAO patients. Increased blood viscosity may play a significant role in the genesis of AAO, which should be determined by prospective randomized studies. 


\section{CONFLICT OF INTEREST}

The authors declare no conflict of interest.

\section{REFERENCES}

1. Crawford JD, Perrone KH, Jung E, et al. Arterial duplex for diagnosis of peripheral arterial emboli. J Vasc Surg. 2016;64:1351-1356. doi: 10.1016/j.jvs.2016.04.005.

2. Engledow AH, Crinnion JN. Acute lower limb ischaemia. Hosp Med. 2002;63:412-415. doi: 10.12968/hosp.2002.63.7.1985.

3. O'Connell JB, Quiñones-Baldrich WJ. Proper evaluation and management of acute embolic versus thrombotic limb ischemia. Semin Vasc Surg. 2009;22:10-16. doi: 10.1053/j. semvascsurg.2008.12.004.

4. Eliason JL, Wainess RM, Proctor MC, et al. A national and single institutional experience in the contemporary treatment of acute lower extremity ischemia. Ann Surg. 2003;238:382389. doi: 10.1097/01.sla.0000086663.49670.d1.

5. Earnshaw JJ, Whitman B, Foy C. National Audit of Thrombolysis for Acute Leg Ischemia (NATALI): clinical factors associated with early outcome. J Vasc Surg. 2004;39:1018-1025. doi: 10.1016/j.jvs.2004.01.019.

6. Lowe G, Lee A, Rumley A, et al. Blood viscosity and risk of cardiovascular events: the Edinburgh Artery Study. Br J Haematol. 1997;96:168-173. doi: 10.1046/j.13652141.1997.8532481.x.

7. Chatzizisis YS, Coskun AU, Jonas M, et al. Role of endothelial shear stress in the natural history of coronary atherosclerosis and vascular remodeling: molecular, cellular, and vascular behavior. J Am Coll Cardiol. 2007;49:2379-2393. doi: 10.1016/j. jacc.2007.02.059.

8. de Simone G, Devereux RB, Chien S, et al. Relation of blood viscosity to demographic and physiologic variables and to cardiovascularrisk factors in apparently normal adults. Circulation. 1990;81:107-117. doi: 10.1161/01.cir.81.1.107.

9. Obara H, Matsubara K, Kitagawa Y. Acute Limb Ischemia. Ann Vasc Dis. 2018;11:443-448. doi: 10.3400/avd.ra.18-00074.

10. Norgren L, Hiatt WR, Dormandy JA, et al. Inter-Society Consensus for the Management of Peripheral Arterial Disease (TASC II). J Vasc Surg. 2007;45:S5-67. doi: 10.1016/j. jvs.2006.12.037.

11. Çekici Y, Kılıç S, Saraçoğlu E, et al. The relationship between blood viscosity and Isolated Coronary Artery Ectasia. Acta Cardiol Sin. 2019;35:20-26. doi: 10.6515/ ACS.201901_35(1).20180701A.

12. Cetin EH, Cetin MS, Canpolat U, et al. Prognostic significance of wholw blood viscosity estimated by de Simone's formula in ST-elevation myocardial infarction. Biomark Med. 2016;10:495-511. doi: 10.2217/bmm.16.10.
13. Cetin MS, Ozcan Cetin EH, et al. The association between whole blood viscosity and coronary collateral circulation in patient with chronic total occlusion. Korean Circ J. 2016;46:784-790. doi: 10.4070/kcj.2016.46.6.784.

14. Ozcan Cetin EH, Cetin MS, Cağlı K, et al. The association of estimated whole blood viscosity with hemodynamic parameters and prognosis in patients with heart failure. Biomark Med. 2019;13:69-82. doi: 10.2217/bmm-2018-0309.

15. Baskurt OK, Yalcin O, Meiselman HJ. Hemorheology and vascular control mechanisms. Clin Hemorheol Microcirc. 2004;30:169-178.

16. Wu KK. Laboratory studies in arterial thromboembolism. In: Koepke JA, ed.Practical Laboratory Hematology. 2nd ed. New York: Churchill Livingstone, 1991; p. 445-467.

17. Trope GE, Lowe GD, McArdle BM, et al. Abnormal blood viscosity and haemostasis in longstanding retinal vein occlusion. Br J Ophthalmol. 1983;67:137-142. doi: 10.1136/ bjo.67.3.137.

18. Gunes $\mathrm{H}$ and Kirisci $\mathrm{M}$. The relationship between whole blood viscosity and deep vein thrombosis. Turkiye Klinikleri J Cardiovasc Sci. 2018;30:6-12. doi: 10.5336/ cardiosci.2018-61921.

19. Taco-Vasquez ED, Barrera F, Serrano-Duenas $M$, et al. Association between blood viscosity and cardiovascular risk factors in patients with arterial hypertension in a high altitude setting. Cureus. 2019;11-e3925. doi: 10.7759/cureus.3925.

20. Sloop GD and Garber DW. The effects of low-density lipoprotein and high-density lipoprotein on blood viscosity correlate with their association with risk of atherosclerosis in humans. Clin Sci (Lond). 1997;92:473-479. doi: 10.1042/ cs0920473.

21. Koenig W, Sund M, Filipiak B, et al. Plasma viscosity and the risk of coronary heart disease:results from the MONICAAugsburg Cohort Study, 1984to 1992. Arterioscler Thromb Vasc Biol. 1998;18:768-772. doi: 10.1161/01.atv.18.5.768.

22. Davies PF. Hemodynamic shear stress and the endotheliumin cardiovascular pathophysiology. Nat Clin Pract Cardiovasc Med. 2009;6:16-26. doi: 10.1038/ncpcardio1397.

23. Naghedi-Baghdar H, Nazari SM, Taghipour A, et al. Effect of diet on blood viscosity in healthy humans: a systematic review. Electron Physician. 2018;10:6563-6570. doi: 10.19082/6563.

24. Saskin H,Ozcan KS, DuzyolC, etal.Areinflammatoryparameters predictors of amputation in acute arterial occlusions? Vascular. 2017;25:170-177. doi: 10.1177/1708538116652995.

25. Lowe G, Rumley A, Norrie J, et al. Blood rheology, cardiovascular risk factors, and cardiovascular disease: the West of Scotland Coronary Prevention Study. Thromb Haemost. 2000;84:553558.

26. Sandhagen B, Lind L. Whole blood viscosity and erythrocyte deformability are related to endothelium-dependent vasodilation and coronary risk in the elderly. Clin Hemorheol Microcirc. 2012;50:301-311. doi: 10.3233/CH-2011-1505. 